\title{
Critical Density for Coverage and Connectivity in Two-Dimensional Fixed-Orientation Directional Sensor Networks Using Continuum Percolation
}

\author{
Mohammad Khanjary a, ${ }^{\text {a, }}$, Masoud Sabaei ${ }^{b}$ and Mohammad Reza Meybodi ${ }^{\text {b }}$
}

\footnotetext{
${ }^{\text {a }}$ Computer and Electrical Engineering Department, Science and Research Branch, Islamic Azad University, Tehran, Iran.

${ }^{\mathrm{b}}$ Computer Engineering and Information Technology Department, Amirkabir University of Technology, Tehran, Iran.
}

\begin{abstract}
Given an initially uncovered field, and as more and more directional sensors (sensors with sector shape sensing area) are continuously added to the sensor network, the size of partial covered areas increases. At some point, the situation abruptly changes from small fragmented covered areas to a single large covered area. This abrupt change is called the sensing-coverage phase transition (SCPT). Likewise, given an originally disconnected sensor network, as more and more sensors are added, the number of connected components changes such that the sensor network suddenly becomes connected. This sudden change is called the network connectivity phase transition (NCPT). Such phase transitions occur in a certain density which is called critical density and finding it is a central topic of Percolation Theory. In this paper, we introduce fixed-orientation directional sensor networks (FIODSNs) and analytically compute critical density of nodes for both SCPT and NCPT in FIODSNs, for all field-of-view angles between 0 and $\pi$ using continuum percolation. In FIODSNs which are the most common type of directional sensor networks, sensor nodes are deployed based on Poisson Point Process, and orientation of them is distributed between 0 and $2 \pi$, independently and uniformly. Due to percolation theory, the critical density is the infimum density for densities above it SCPT and NCPT almost surely occur. Therefore, the results could be used to prepare coverage and connectivity in FIODSNs. Moreover, to solve the SCPT and NCPT problems together, we propose a model for percolation in directional sensor networks which could be used in other related researches.
\end{abstract}

Keywords: Critical density, continuum percolation, sensing coverage, network connectivity, fixedorientation directional sensor networks, video sensor networks, FIODSN.

\section{Introduction}

Sensing coverage can be considered as one of the main criterion of quality of service in sensor networks. Extensive researches have been done to solve technical problems related to coverage in sensor networks and several types of coverage have been introduced by researchers so far. Network connectivity, on the other hand, is a graph-theoretic concept that helps sensors communicate with each other for forwarding their data to a central gathering node, called the sink. To sense a region of interest sufficiently and receive the sensed data by the sink, it is necessary that both sensing coverage and network connectivity be maintained. Due to wide range of technical problems in sensing coverage and network connectivity and

Submitterd: October 15, 2014; Revised: May 31, 2015; Accepted: August 8, 2015.

* Corresponding author.

E- mail addresses: khanjary@ srbiau.ac.ir (M. Khanjary), sabaei@aut.ac.ir (M. Sabaei), mmeybodi@ aut.ac.ir (M. R. Meybodi). 
also their significance, a lot of researches have been focused on them. Some recent reviews on different issues of coverage and connectivity in general sensor networks could be found in [1-3] and in directional sensor networks could be found in [4-5].

One of the main challenges about coverage and connectivity in sensor networks, is finding a certain density that coverage and connectivity almost surely occur for densities above it. Finding such densities is the main topic in Percolation Theory. In other word, the main topic in Continuum Percolation is finding the critical density of a Poisson point process at which an unbounded covered/connected component almost surely appears in the network. Percolation Theory prepares a rigorous mathematical model for assessing coverage and connectivity in wireless networks and it has been used in several recent researches e.g. [8] and [23]. In this paper, we use Continuum Percolation to analytically compute the critical density for coverage and connectivity in fixed-orientation directional sensor networks.

\subsection{Problem Statement}

Finding the minimum number of sensors required to achieve a certain degree of sensing coverage and network connectivity is one of the fundamental issues in design of sensor networks. This will be more important and complicated when we deal with directional sensor networks. As more and more directional sensors are continuously deployed, the size of covered areas increases and, at some instant, the situation suddenly changes from small fragmented covered areas to a single large covered area which spans the entire field. We call this sudden change in the sensing coverage of a field the sensing-coverage phase transition (SCPT). Likewise, the number of connected components increases when more and more directional sensors are continuously added to a sensor network that is originally disconnected. At some point, the situation abruptly changes from a disconnected network to connected network. We call this abrupt change in the network topology the network-connectivity phase transition (NCPT).

Because these phase transitions occur at a given density (called critical density) and study of such phase transitions is an important topic in percolation theory, we are interested in finding the critical density for SCPT and NCPT and then finding critical density for both by using percolation theory. Due to percolation theory, the critical density is infimum density that for densities above it SCPT and NCPT almost surely occur [8].

Based on orientation properties of sensor nodes, we classified directional sensor networks to three categories: 1) Aligned-orientation directional sensor networks (ALODSNs) in which the orientation of all sensor nodes is the same and fixed. 2) Fixed-orientation directional sensor networks (FIODSNs) in which orientation of nodes is distributed on $[0,2 \pi]$ independently and uniformly and is fixed. 3) Adjustableorientation directional sensor networks (ADODSNs) in which the deployment is such as FIODSNs but the orientation of sensing sectors could be adjusted after deployment by using an algorithm. In [8], we analytically proposed a general approach to calculate the critical density for sensing coverage and network connectivity in ALODSNs for different field-of-view angles between 0 and $\pi$ by using continuum percolation and in this paper, we present an approach to calculate the critical density in FIODSNs.

\subsection{Related works}

\subsubsection{Density of Nodes in Directional Sensor Networks}

While most of researches in this subject focused on placement e.g. [9], Target Tracking e.g. [10], scheduling e.g. [11-12] and k-angel coverage e.g. [13], there are a few researches on density of nodes in directional sensor networks.

In [14], Liu et al. first proposed a new notion of coverage, entitled localization-oriented coverage (Lcoverage for short) by using Bayesian estimation theory and then analyzed the relationship between the density of camera sensors and the L-coverage probability under random deployment. In [15], Han et al. considered the problems of deploying a minimum number of directional sensors to form a connected network to cover either a set of point locations or the entire target sensing area which are NP-hard and then 
presented two approximation algorithms for them. In [16], Chen et al. proposed a weighted centralized greedy algorithm (WCGA) to assign a weight to each orientation of each sensor. The weight function added in the WCGA could be adjusted according to different density of sensor nodes in directional sensor networks. Then, each sensor will select its orientation which has highest weight.

In [17], Wang et al. proposed a novel model called full-view coverage. An object is considered to be fullview covered if for any direction from 0 to $2 \pi$ (object's facing direction), there is always a sensor such that the object is within the sensor's range and more importantly the sensor's viewing direction is sufficiently close to the object's facing direction. They also derived a sufficient condition on the sensor density needed for full-view coverage in a random uniform deployment.

\subsubsection{Percolation Theory in Sensor Networks}

The concept of continuum percolation, originally due to Gilbert [18], is to find the critical density of a Poisson point process at which an unbounded connected component almost surely appears so that the network can provide long-distance multihop communication. Since then, Gilbert's model has become the basis for studying continuum percolation in wireless networks e.g. [19-20]. Recently, percolation theory has been considered by researchers to be used to examine coverage and connectivity in sensor networks too [6-7] and [21-26].

Ammari et al. [6] considered the critical density required to prepare sensing coverage and network connectivity in sensor networks simultaneously by using continuum percolation in two-dimensional sensor networks. Then, they extended their research to three-dimensional sensor networks [7]. Xing et al. [21] considered the proper time to redeployment of failed nodes to keep the network connected. They examined the time of first partition in network and found it must be between $\log (\log n)$ and $(\log n)^{1 / p}$ which $n$ is the network size and $\rho>1$. Liu et al. [22-23] considered exposure-path prevention. Exposure-path refers to the path in a sensor network that an intruder could traverse and not being detected by sensor nodes. They proposed a bond-percolation theory based scheme by mapping the exposure path problem into a bond percolation model. Using this model, they derived the critical densities for omnidirectional sensor networks and directional sensor networks [24]. Yang et al. [25] considered following problem: given a randomly deployed sensor network where sensors are active with probability $p$, how many sensors are needed to achieve connected-k-coverage? Connected-k-coverage requires the monitored region to be k-covered by a connected component of active sensors, which is less demanding than traditional k-coverage and connectivity in which all active sensors participate in both coverage and connectivity simultaneously. They investigated the theoretical foundations about connected-k-coverage by applying the percolation theory and derived the critical density for connected-k-coverage under different ratio between sensing and communication radius of sensors.

Also, Balister et al. [26] introduced a new type of coverage called trap coverage that scales better with large deployment regions. A sensor network providing trap coverage guarantees that any moving object or phenomena can move at most a known displacement before to be detected by the network, for any trajectory and speed. Also, they proposed some polynomial-time algorithms to determine the level of trap coverage achieved once sensors are deployed on the ground.

The research of this paper is similar to research done by Ammari et al. [6-7] for omnidirectional sensor networks. But we are interested to do such research when the sensor network consists of nodes with only directional sensing ability such as camera sensor networks and Doppler probes.

\subsection{Contribution}

The contributions of this paper are two folds:

1. In this paper, we consider the directional sensor networks in which sensor nodes have been deployed in the region of interest according to Poisson point process and the orientation of sensor nodes distributed on $[0,2 \pi]$ uniformly and independently. While most of researches in this field are based 
on Monte Carlo method [27], we present a general approach to find the critical density analytically based on field-of-view angle of sensors between 0 and $\pi$. To the best of our knowledge, this is the first attempt to find the critical density for sensing coverage and network connectivity in such networks. Due to random nature of directional sensor networks and increasing interest to directional sensor networks such as video/camera sensor networks, this research could be used for practical deployments.

2. Also, we propose a concentric disk and sector model for percolation in directional sensor networks which provides a basis for solving the SCPT and NCPT problems together.

The remainder of this paper is organized as follows: section 2 presents the model and definitions of the paper, section 3 presents our method for calculation of critical density for sensing coverage in FIODSNs, section 4 discusses on network connectivity in FIODSNs, Section 5 discusses on integrated sensing coverage and network connectivity in FIODSNs, section 6 presents the simulation results for examining the calculated critical densities and finally section 7 concludes the paper and presents the possible future works.

\section{Model and Terminology}

\subsection{Definitions}

This section defines the terms we will use throughout the paper and the network and percolation model.

Definition 1 (spatial Poisson point process) [28]. Let $X_{\lambda}$ be a random variable representing the number of points in an area $A$. The probability that there are $k$ points inside $A$ is computed as

$$
P\left(X_{\lambda}(A)=k\right)=\frac{\lambda^{k}|A|^{k}}{k !} e^{-\lambda|A|}
$$

for all $k \geq 0$, where $|A|$ is the area of $A$ and $\lambda$ is the density per unit area. In this paper, center of sensor nodes $\left(\xi_{i}\right)$ is considered as points in Poisson point process.

Definition 2 (directional sensor nodes). Each sensor node $i$ is denoted by a tuple $S_{i}\left(\xi_{i}, r, \varphi, \vec{\vartheta}_{l}, R\right)$ where $\xi_{i}$ is the center, $r$ is the sensing radius, $\varphi$ is the field-of-view angle $(0<\varphi \leq$ $\pi), \overrightarrow{\vartheta_{l}}$ is the orientation vector and $R$ is the transmission radius of the sensor.

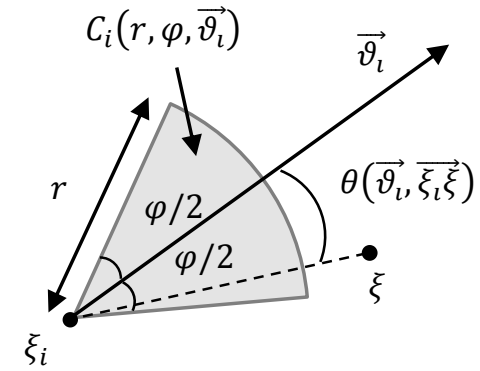

(a)

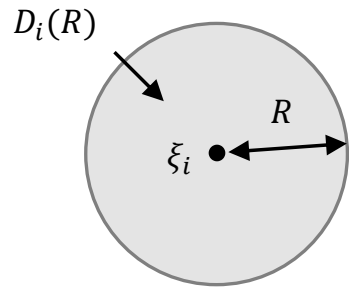

(b)

Fig. 1. (a) Sensing range (b) Transmission range. 


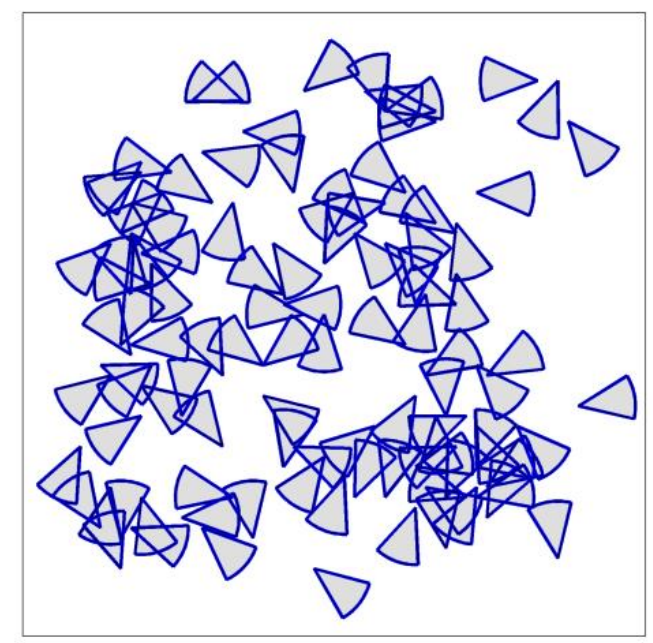

Fig. 2. A fixed-orientation directional sensor network (FIODSN) with $\varphi=\pi / 4$.

Definition 3 (sensing range). The sensing range of a directional sensor node $S_{i}\left(\xi_{i}, r, \varphi, \overrightarrow{\vartheta_{l}}, R\right)$ is a sector which defined by

$$
C_{i}\left(r, \varphi, \overrightarrow{\vartheta_{l}}\right)=\left\{\xi \in \mathbb{R}^{2}:\left|\xi_{i}-\xi\right| \leq r \text { and } \theta\left(\overrightarrow{\vartheta_{l}}, \overrightarrow{\xi_{l}} \xi\right) \leq \frac{\varphi}{2}\right\}
$$

where $\left|\xi_{i}-\xi\right|$ stands for the Euclidean distance between $\xi_{i}$ and $\xi, \theta\left(\overrightarrow{\vartheta_{l}}, \overrightarrow{\xi_{l} \xi}\right)$ stands for angle between orientation vector of sensor $\left(\overrightarrow{\vartheta_{l}}\right)$ and vector from point $\xi_{i}$ to $\xi$ (see Fig. 1 (a)).

Definition 4 (transmission range). The transmission range of sensor $S_{i}\left(\xi_{i}, r, \varphi, \overrightarrow{\vartheta_{l}}, R\right)$ is a disk of radius $R$ which defined by

$$
D_{i}(R)=\left\{\xi \in \mathbb{R}^{2}:\left|\xi_{i}-\xi\right| \leq R\right\}
$$

where $\left|\xi_{i}-\xi\right|$ stands for the Euclidean distance between $\xi_{i}$ and $\xi$ (see Fig. 1 (b)).

Definition 5 (Fixed-Orientation Directional Sensor Network (FIODSN)). A FIODSN is a directional sensor network in which orientation of sensors is distributed on $[0,2 \pi]$ uniformly and independently. In FIODSNs, the orientation of sensors is fixed and unchangeable (see Fig. 2)

Definition 6 (collaborating sensors). Two sensors $S_{i}$ and $S_{j}$ are said to be collaborating if and only if there exist some points belong to sensing range of both sensors (see Fig. 3 (b)).

$$
\operatorname{Col}\left(S_{i}\right)=\left\{S_{j}: \exists \xi \in \mathbb{R}^{2}, \xi \in C_{i} \text { and } \xi \in C_{j}\right\}
$$

Definition 7 (communicating sensors). Two sensors $S_{i}$ and $S_{j}$ are said to be communicating if and only if the Euclidean distance between the centers of their transmission disks is less than $R$ (see Fig. 3 (a)).

$$
\operatorname{Com}\left(S_{i}\right)=\left\{S_{j}:\left|\xi_{i}-\xi_{j}\right| \leq R\right\}
$$




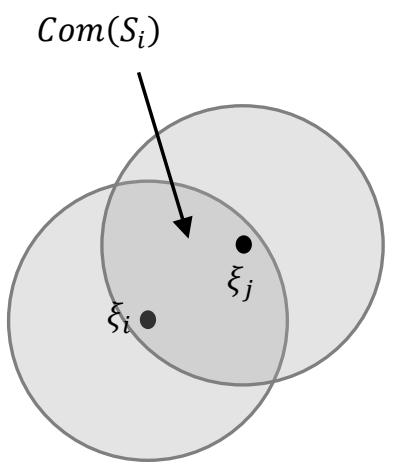

(a)

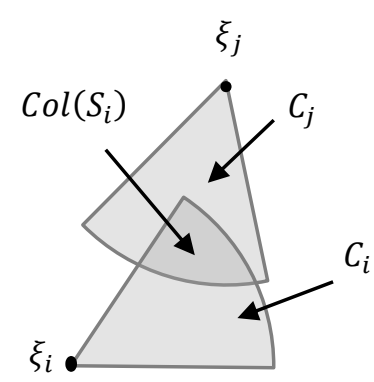

(b)

Fig. 3. (a) Communicating sensors (b) Collaborating sensors.

Definition 8 (filling factor) [29]. If each object has area equal to $a$, the filling factor of a homogenous Poisson point process with density equal to $\lambda\left(X_{\lambda}=\left\{\xi_{i}: i \geq 1\right\}\right)$ is given by

$$
\phi=1-e^{-\eta}
$$

which is the mean fraction of region covered by the objects. In equation (2), $\eta$ is the density of objects and is given by

$$
\eta=\lambda a
$$

where $a$ is the area of objects and $\lambda$ is the density of the Poisson point process.

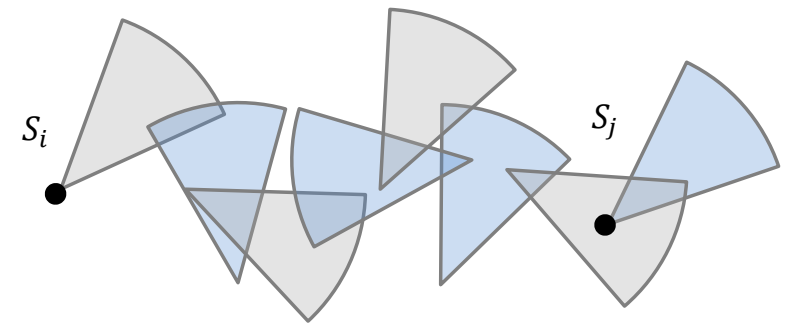

(a)

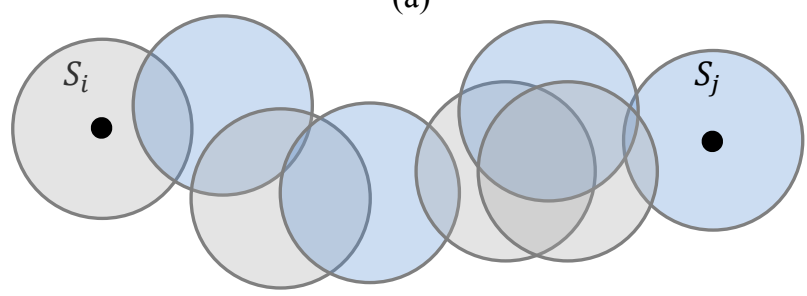

(b)

Fig. 4. (a) Collaboration path and (b) Communication path between sensors $S_{i}$ and $S_{j}$.

In this paper, like other researches in percolation theory e.g. [6-7] and [27], we are interested in finding $\eta$ and $\phi$ in FIODSNs rather than $\lambda$, because they are independent of sensing radius $(r)$ and communication radius $(R)$ (in general the area of the objects) and also could be used to compare with other researches.

Definition 9 (collaboration and communication path). A collaboration path between two sensors $S_{i}$ and $S_{j}$ is a sequence of sensors $S_{i}, S_{i+1}, \ldots, S_{j-1}, S_{j}$ such that any pair of sensors $S_{k}$ and $S_{k+1}$, for $i \leq k \leq j-$ 1, are collaborating (see Fig. 4 (a)). A communication path between two sensors $S_{i}$ and $S_{j}$ is a sequence of 
sensors $S_{i}, S_{i+1}, \ldots, S_{j-1}, S_{j}$ such that any pair of sensors $S_{k}$ and $S_{k+1}$, for $i \leq k \leq j-1$, are communicating (see Fig. 4 (b)).

Definition 10 (covered k-component). A set of sensing sectors $\left\{C_{i}\left(r, \varphi, \vec{\vartheta}_{i}\right): 1 \leq i \leq m\right\}$ is said to be a covered component if and only if it is maximal and there exists a collaboration path between any pair of sensors $S_{i}$ and $S_{j}$, for all $1 \leq j, k \leq m$, and $j \neq k$. A covered k-component, denoted by $C_{K}^{C O V}$, is a covered component having k sensing sectors.

Definition 11 (connected k-component). A set of transmission disks $\left\{D_{i}(R): 1 \leq i \leq n\right\}$ is said to be a connected component if and only if it is maximal and there exists a communication path between any pair of sensors $S_{i}$ and $S_{j}$, for all $1 \leq j, k \leq n$, and $j \neq k$. A connected k-component, denoted by $C_{K}^{C O N}$, is a connected component having $\mathrm{k}$ transmission disks.

Definition 12 (base circle). The base circle for an object is a circle of radius $\gamma$ in which if $i \geq 1$ objects completely put inside of it, then the objects will overlap each other regardless of the number of objects. In other word, the objects inside the base circle form a complete graph in which each object overlap other objects.

In fact, the base circle is the basic form of clusters in percolation. Radius of base circle is dependent to the size and form of objects and finding it for directional sensors is one of our steps to find the critical density. In [6], it has been shown that circle is the most compressed form for base cluster and assuming other forms such as ellipse instead of circle will return same result.

Definition 13 (probability of a k-component) [6]. If the objects are distributed in the region based on a homogenous Poisson point process, the probability of existence a covered/connected k-component in a circle is given by

$$
P(k)=\frac{\left(9 \lambda \pi R_{k}^{2} / 4\right)^{k}}{k !} e^{-\lambda \pi R_{k}^{2}}
$$

where $R_{k}$ is radius of the circle, $k$ is the number of objects in the component and $\lambda$ is the density of Poisson point process.

\subsection{Percolation Theory}

Due to Broadbent and Hammersley [30] percolation model gave birth as a model for disordered mediums. In general, percolation theory is divided to two models called discrete percolation [31] and continuum percolation [28].

In discrete percolation (also called the lattice model), the sites of the lattice are close or open due to probability $p$ and may have different tessellation such as square, triangle, honeycomb and etc. While in continuum percolation, the positions of the sites are randomly distributed and thus, there is no need to have a different analysis for each of these regular lattices. While in discrete percolation, we are interested in finding the critical probability denoted by $p_{c}$ in which percolation occurs, in continuum percolation we are interested in finding the critical density denoted by $\lambda_{c}$ at which an infinite or large clump of overlapping objects first appears that spans the entire network. The density $\lambda_{c}$ is the critical value for the density $\lambda$ such that there exists no such clump of overlapping objects almost surely when $\lambda<\lambda_{c}$ (the system is said to be in the subcritical phase), but it exists almost surely when $\lambda>\lambda_{c}$ (the system is said to be supercritical) and we say that percolation occurs.

In this paper, we consider a continuum percolation model which consists of homogeneous points (representing center of the sensors) that are randomly distributed in $\mathbb{R}^{2}$ according to a spatial Poisson point process of density $\lambda$. 


\subsection{Percolation Model}

A percolation model can be viewed as an ensemble of points distributed in space, where some pairs are connected [32]. We consider a Boolean model [28] which is defined by two components, namely, point process $X_{\lambda}$ and connection function $h$. The set $X_{\lambda}=\left\{\xi_{i}: i \geq 1\right\}$ is a homogeneous Poisson point process of density $\lambda$ in a two dimensional Euclidean plane $\mathbb{R}^{2}$, where the elements of $X_{\lambda}$ are the locations of the sensors used to cover a field. The connection function $h$ is defined such that two points $\xi_{i}$ and $\xi_{j}$ are adjacent independently of all other points with probability given by

$$
h_{\text {CoV }}\left(\xi_{i}, \xi_{j}\right)= \begin{cases}1 & \text { if } \exists \xi \mid \xi \in C_{i} \text { and } \xi \in C_{j} \\ 0 & \text { otherwise, }\end{cases}
$$

for sensing coverage and given by

$$
h_{\text {CON }}\left(\xi_{i}, \xi_{j}\right)= \begin{cases}1 & \text { if }\left|\xi_{i}-\xi_{j}\right| \leq d \\ 0 & \text { otherwise }\end{cases}
$$

for network connectivity, where $\left|\xi_{i}-\xi_{j}\right|$ is the Euclidean distance between $\xi_{i}$ and $\xi_{j}$.

\section{Critical Density for Sensing Coverage in FIODSNs}

Let $X_{\lambda}=\left\{\xi_{i}: i \geq 1\right\}$ be a two-dimensional homogeneous Poisson point process with density of $\lambda$ (the number of sensors per unit area) and connection function $h_{C O V}$ (equation (5), where $\xi_{i}$ represents the location of the sensor $S_{i}$.

\subsection{Asymmetry of Sensing Range in Directional Sensors}

As it is shown in Fig. 5, the sensing range of sensors in FIODSNs (in general, in directional sensors) is a circular sector. In Fig. $5, l(\varphi, r)$ is the length of front side of sensing sector and could be calculated by using law of cosine [33].

$$
\begin{aligned}
& l(\varphi, r)^{2}=r^{2}+r^{2}-2 r^{2} \cos (\varphi) \\
& l(\varphi, r)=\sqrt{2 r^{2}(1-\cos (\varphi))} \\
& l(\varphi, r)=r \sqrt{2(1-\cos (\varphi))}
\end{aligned}
$$

where $r$ is the radius of sensing sector and $\varphi$ is the field-of-view angle.

As it is seen in Fig. 5, sectors have asymmetry. They have vertical or horizontal elongation. To find this elongation, we assume that if we want to put the sector in a rectangle, which side will be the longest side of this rectangle. This will help us to find the length of biggest gap could be filled by sensors. As seen, the height of rectangle when $\varphi \in(\pi / 3, \pi]$ is higher than $r$ and therefore, is the longest side of rectangle. However, when $\varphi \in(0, \pi / 3]$ the height of rectangle is less than or equal to $r$. Therefore, the longest side of rectangle is its width and its length is equal to $r$. Therefore, as a general equation, the longest side of the rectangle which represents the length of biggest gap could be filled by sensors is given by

$$
l_{\max }(\varphi, r)= \begin{cases}r \sqrt{2(1-\cos (\varphi))} & \text { if } \varphi \in\left(\frac{\pi}{3}, \pi\right] \\ r & \text { if } \varphi \in\left(0, \frac{\pi}{3}\right]\end{cases}
$$

Table 1 shows $l(\varphi, r)$ and $l_{\max }(\varphi, r)$ of sectors for eight different field-of-view angles. 


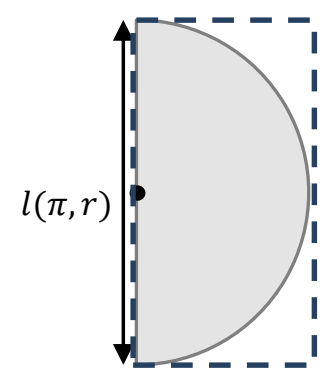

(a)

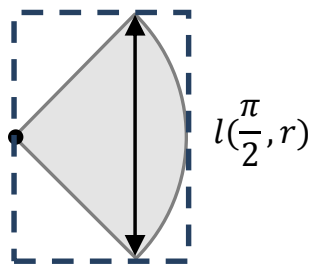

(c)

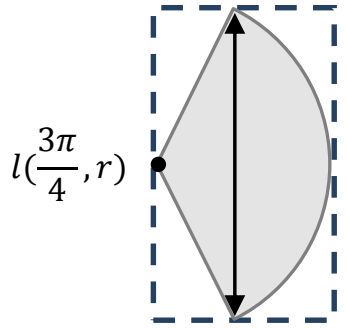

(b)

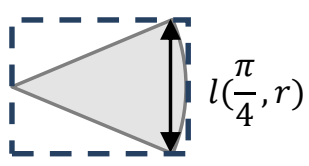

(d)

Fig. 5. $1(\varphi, r)$ for sensors with (a) $\varphi=\pi$, (b) $\varphi=3 \pi / 4$, (c) $\varphi=\pi / 2$, and (d) $\varphi=\pi / 4$.

\subsection{Form of Clusters}

It has been shown in [6] that circle is the most compressed form for clusters of sensors in percolation and it has been used for calculations. However, it also has been shown that the calculations will be correct for other forms such as ellipse. Therefore, we use this form of clusters for our calculations too. As an instance, a cluster of directional sensors with field-of-view angle equal to $\pi$ is shown in Fig. 6 where $R_{k}$ is the radius of cluster and $\xi\left(C_{k}^{\text {cov }}\right)$ is the center of cluster.

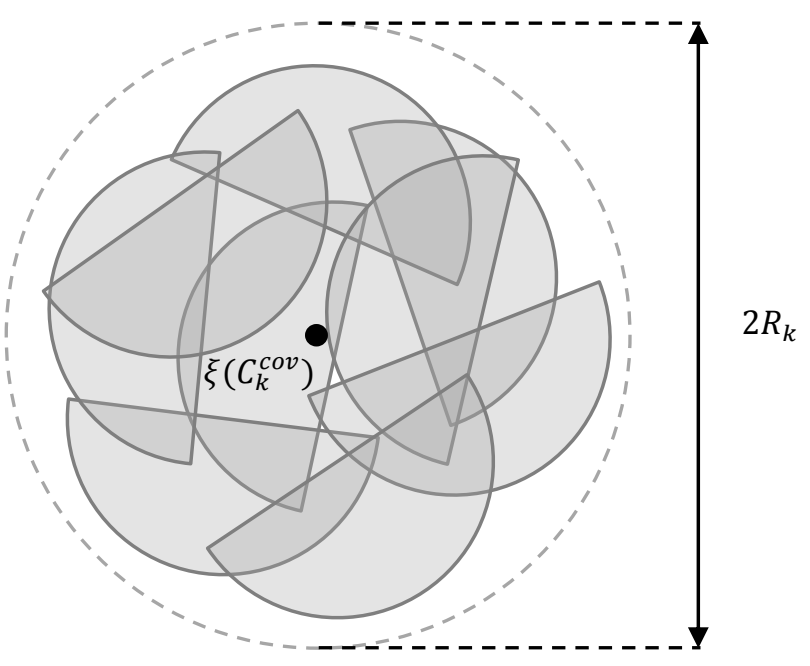

Fig. 6. A cluster for directional sensors with $\varphi=\pi$. 
Table 1

Elongation in Circular Sectors for 8 Different $\varphi$

\begin{tabular}{ccc}
\hline \hline$\varphi$ & $\begin{array}{c}l(\varphi, r) \\
(\times r)\end{array}$ & $\begin{array}{c}l_{\max }(\varphi, r) \\
(\times r)\end{array}$ \\
\hline$\pi$ & 2.00000 & 2.0000 \\
$3 \pi / 4$ & 1.84776 & 1.8478 \\
$2 \pi / 3$ & 1.73205 & 1.7321 \\
$\pi / 2$ & 1.41421 & 1.4142 \\
$\pi / 3$ & 1.00000 & 1.0000 \\
$\pi / 4$ & 0.76537 & 1.0000 \\
$\pi / 6$ & 0.51764 & 1.0000 \\
$\pi / 12$ & 0.26105 & 1.0000 \\
\hline \hline
\end{tabular}

\subsection{Analytical Calculations}

In this section, we present several lemmas and theorems to compute the critical density for SCPT based on field-of-view angle $(\varphi)$ of sensors. The approach of calculations can be expressed as follows: First, we will present two equations for average distance between centers of clusters in percolation (Lemma 1 and 2) and then derive an equation for density of clusters by equating them (Theorem 1). Then, we will present another equation to find the density of clusters by using the probabilistic nature of Poisson point process (Theorem 2). Finally, we derive the critical density of nodes (and the filling factor) by equating two found equation for density of clusters (Theorem 1 and 2).

\subsubsection{Critical Density of Covered Components}

Although there exist a few definitions of the average distance between clusters (covered components in our case), one of them is more appropriate. It is defined as the average of the minimum distance between all pairs of sensing disks, each from one covered component. Lemma 1 computes the mean distance between neighboring covered k-components at critical percolation. It is noticed that to find a general equation for sensors with different field-of-view angles, we will represent the distances as $\alpha \mathrm{r}$ and present a general approach to find the coefficient $\alpha$ for different field-of-view angle in next section.

Lemma 1. Let $\left\{C_{k}^{c o v}\right\}$ be a set of covered k-components with density $\lambda(k)$ and $Y$ a random variable representing distances between them. The mean distance $d_{a v g}^{1}$ between two neighboring covered components at critical percolation is given by

$$
d_{a v g}^{1}=\frac{1}{2 \sqrt{\lambda_{c}(k)}}
$$

where $\lambda_{c}(k)$ is the density of $\left\{C_{k}^{c o v}\right\}$ at critical percolation.

Proof. See [6], page 1428.

Lemma 2 computes the average distance between neighboring covered k-components at critical percolation using another approach.

Lemma 2. Let $\left\{C_{k}^{c o v}\right\}$ be a set of covered k-components with density $\lambda(k)$ and $Y$ a random variable representing distances between them. The mean distance $d_{\text {avg }}^{2}$ between two neighboring covered components at critical percolation is given by 


$$
d_{\text {avg }}^{2}=\frac{\operatorname{erf}\left(\alpha \sqrt{\lambda_{c} \pi} r\right)-\alpha^{2} \sqrt{\lambda_{c}} r e^{-\alpha^{2} \lambda_{c} \pi r^{2}}}{2 \sqrt{\lambda_{c}}}
$$

where $\alpha r$ is the distance of covered k-component and $\lambda_{c}$ is the density of a set of sensing sectors $\left\{C_{i}\left(r, \varphi, \vec{\vartheta}_{i}\right): i \geq 1\right\}$ at critical percolation.

Proof. For a homogeneous Poisson point process, the probability that there is no neighbor within distance $\sigma$ of an arbitrary point is given by $e^{-\lambda \pi \sigma^{2}}$ [34]. Therefore, the probability that the distance between a point and its neighbor is less than or equal to $\sigma$ is equal to

$$
P[Y \leq \sigma]=1-e^{-\lambda \pi \sigma^{2}}
$$

Hence, the corresponding probability density function is given by

$$
f(Y \mid Y \leq \sigma)=2 \lambda \pi \sigma e^{-\lambda \pi \sigma^{2}}
$$

The mean distance $d_{\text {avg }}^{2}$ between two neighboring covered k-components of $\left\{C_{k}^{c o v}\right\}$ at critical percolation is obtained when the distance $\sigma$ between two sensing sectors, say $C_{i}\left(r, \varphi, \vec{\vartheta}_{i}\right)$ and $C_{j}\left(r, \varphi, \vec{\vartheta}_{j}\right)$, each from one covered component, belongs to the interval $[0, \alpha r]$. Therefore,

$$
d_{\text {avg }}^{2}=E[Y \mid Y \leq \alpha r]=\int_{0}^{\alpha r} \sigma \times f(Y \mid Y \leq \sigma) d \sigma=\frac{e r f\left(\alpha \sqrt{\lambda_{c} \pi} r\right)-\alpha^{2} \sqrt{\lambda_{c}} r e^{-\alpha^{2} \lambda_{c} \pi r^{2}}}{2 \sqrt{\lambda_{c}}}
$$

where $\operatorname{erf}(x)$ is the error function [35] and is given by

$$
\operatorname{erf}(x)=\frac{2}{\sqrt{\pi}} \int_{0}^{x} e^{-t^{2}} d t
$$

By using Lemmas 1 and 2, Theorem 1 computes the density of covered k-components at critical percolation.

Theorem 1. The critical density of a set of covered k-components $\left\{C_{k}^{c o v}\right\}$ is given by

$$
\lambda_{c}(k)=\frac{\lambda_{c}}{\left(\operatorname{erf}\left(\alpha \sqrt{\lambda_{c} \pi} r\right)-\alpha^{2} \sqrt{\lambda_{c}} r e^{-\alpha^{2} \lambda_{c} \pi r^{2}}\right)^{2}}
$$

where $\alpha r$ is the distance of covered k-component and $\lambda_{c}$ is the density of sensing sectors at critical percolation and, $\operatorname{erf}(x)$ is the error function [35].

Proof. From Lemma 1, equation (9) and Lemma 2, equation (10), the mean distance between two covered $\mathrm{k}$-components at critical percolation should verify the equality $d_{a v g}^{1}=d_{a v g}^{2}$. It follows that the density of covered k-components, $\lambda_{c}(k)$, at critical percolation is given by

$$
\lambda_{c}(k)=\frac{\lambda_{c}}{\left(\operatorname{erf}\left(\alpha \sqrt{\lambda_{c} \pi} r\right)-\alpha^{2} \sqrt{\lambda_{c}} r e^{-\alpha^{2} \lambda_{c} \pi r^{2}}\right)^{2}}
$$




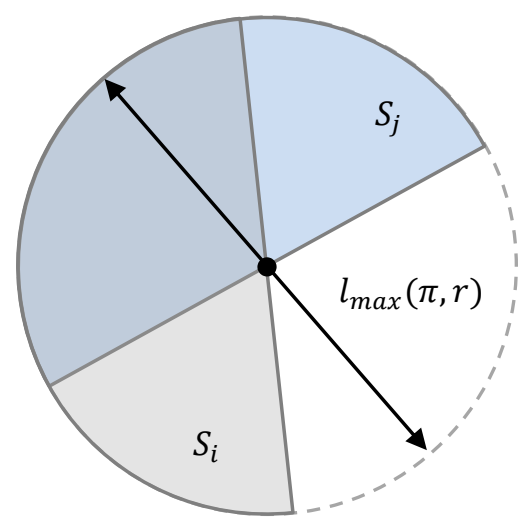

Fig. 7. Base circle for sensors with field-of-view angle $\varphi=\pi$.

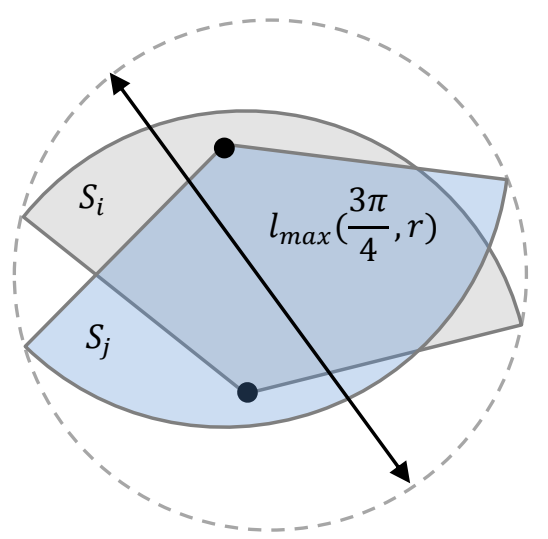

Fig. 8. Base circle for sensors with field-of-view angle $\varphi=3 \pi / 4$.

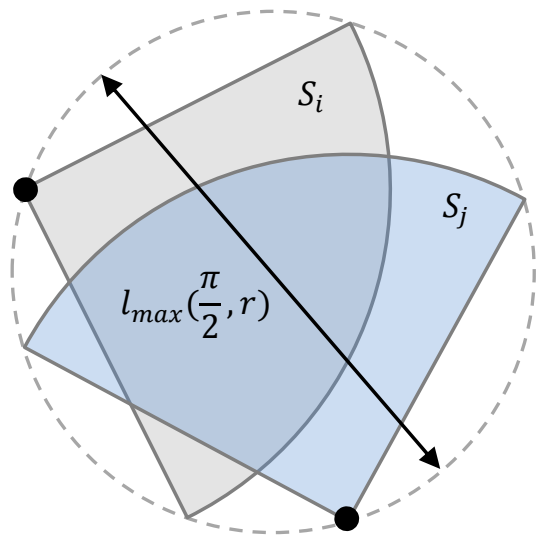

Fig. 9. Base circle for sensors with field-of-view angle $\varphi=\pi / 2$. 


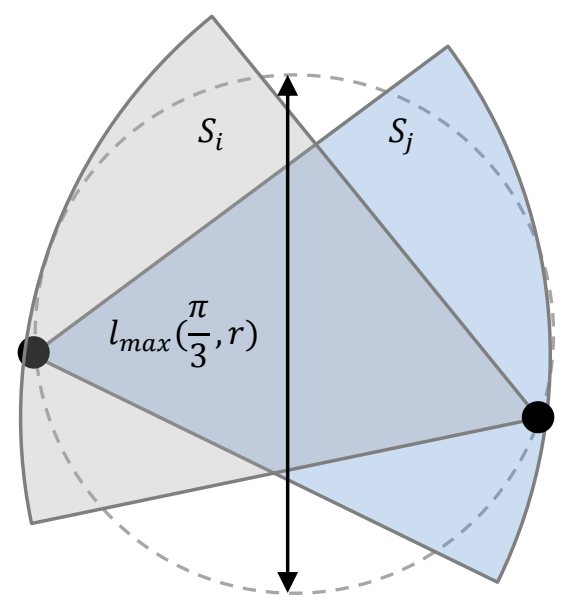

Fig. 10. Problem of sensors with field-of-view angle $\varphi=\pi / 3$. As it is seen $l_{\max }(\varphi, r)$ could not be used as the radius of base circle.

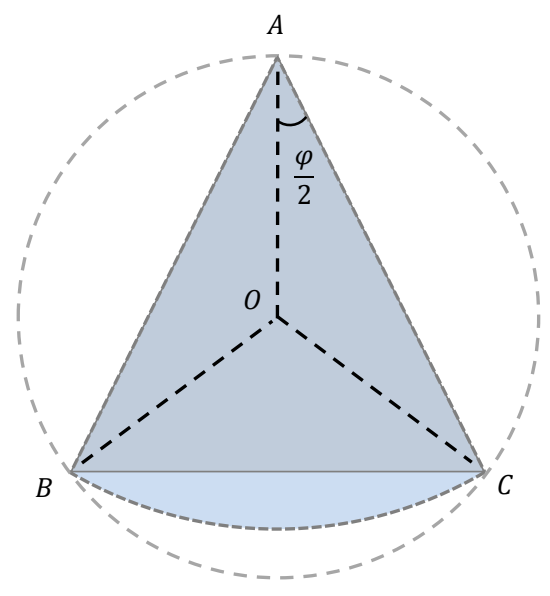

Fig. 11. General form for calculation of radius of base circle for sensor with field-of-view angle less than $\pi / 2$.

\subsubsection{Base Circles}

As it is expressed in definition 12, the base circle for an object is the circle of radius $\gamma$ in which if all area of objects put inside of it, then the objects will overlap each other regardless of number of objects. In this section, we present a general approach to calculate the radius of base circle for sensors based on their fieldof-view angles.

As it is shown in section 3.1 and according to table 1, the longest side of a circumference rectangle for a sector could be found by equation (8). Therefore, it could help us to find the radius of base circles.

The base circle for $\varphi=\pi, 3 \pi / 4$ and $\pi / 2$ are shown in Fig. 7, Fig. 8 and Fig. 9, respectively. In these figures, the diameter is equal to $\mathrm{l}_{\max }(\varphi, \mathrm{r})$ and therefore the radius of their base circle is its half. But as it is seen in Fig. 10, for $\varphi \leq \pi / 2$, all part of sectors could not be included in the circle with diameter equal to $l_{\max }(\varphi, r)$. To solve this problem, we use the law of cosine [33] again. Consider Fig. 11, as a general form of base circles for sectors with field-of-view angles less than or equal to $\pi / 2$. We are interested in finding the radius of circle $(\overline{O A}$ or $\overline{O B}$ or $\overline{O C})$. Considering triangle $\triangle A O C$ and by using law of cosine for angle $\angle O A C$ we have 


$$
O C^{2}=O A^{2}+A C^{2}-2 \times O A \times A C \times \cos \left(\frac{\varphi}{2}\right)
$$

where $\overline{O C}$ is the line segment between $O$ and $C$ and $O C$ is its length. Because $O C=O A$, we can simplify the equation to

$$
\begin{gathered}
2 \times O A \times A C \times \cos \left(\frac{\varphi}{2}\right)=A C^{2} \\
O A=\frac{A C}{2 \times \cos \left(\frac{\varphi}{2}\right)}
\end{gathered}
$$

Since $A C$ is equal the radius of the sensing sector $(r)$, the radius of base circle $(O A)$ could be rewritten as

$$
O A=\frac{r}{2 \times \cos \left(\frac{\varphi}{2}\right)}
$$

Finally, by combination of equations (8) and (12), we can derive a general equation for radius of base circle for sectors with field-of-view angles between 0 and $\pi$.

$$
t(\varphi, r)= \begin{cases}\frac{r \sqrt{2(1-\cos (\varphi))}}{2} & \frac{\pi}{2}<\varphi \leq \pi \\ \frac{r}{2 \cos \left(\frac{\varphi}{2}\right)} & 0<\varphi \leq \frac{\pi}{2}\end{cases}
$$

By dividing equation (13) to $r$, the coefficient $\alpha$ of equation (11) and equations of next section will be found.

$$
\alpha(\varphi)=\frac{t(\varphi, r)}{r}= \begin{cases}\frac{\sqrt{2(1-\cos (\varphi))}}{2} & \frac{\pi}{2}<\varphi \leq \pi \\ \frac{1}{2 \cos \left(\frac{\varphi}{2}\right)} & 0<\varphi \leq \frac{\pi}{2}\end{cases}
$$

Table 2 shows the $\alpha(\varphi)$ for eight angles of table 1 .

Table 2

Radius of Base Circle $(\alpha)$ for 8 different $\varphi$

\begin{tabular}{cc}
\hline \hline$\varphi$ & $\alpha(\varphi)$ \\
\hline$\pi$ & 1.0 \\
$3 \pi / 4$ & 0.9239 \\
$2 \pi / 3$ & 0.8660 \\
$\pi / 2$ & 0.7071 \\
$\pi / 3$ & 0.5774 \\
$\pi / 4$ & 0.5412 \\
$\pi / 6$ & 0.5176 \\
$\pi / 12$ & 0.5043 \\
\hline \hline
\end{tabular}

\subsubsection{Critical Radius of Covered Components}

As it is said in previous section, there is a particular value of the radius $\mathrm{R}_{\mathrm{k}}$ of the circular shape enclosing 
a covered component that almost surely guarantees the formation of special class of covered k-components, called critical covered k-components. Any nonempty circle of radius $\alpha \mathrm{r}$ should enclose a covered kcomponent. In other words, regardless of the number of sensing sectors of radius $r$ and field-of-view angle $\varphi$ located in a circle of radius $\alpha r$, these sensing sectors should definitely form a covered k-component. Moreover, this covered $\mathrm{k}$-component is a complete graph in that each pair of sensors, say $\mathrm{S}_{\mathrm{i}}$ and $\mathrm{S}_{\mathrm{j}}$, whose sensing sectors are totally included inside this circle of radius $\alpha \mathrm{r}$ are collaborating. Due to definition 12, we called these particular circles as base circles and presented a general equation to find their radius.

Theorem 2 computes the density of critical covered k-components at critical percolation.

Theorem 2. At critical percolation, the density of covered k-components, which are enclosed in circles whose radii is equal to $\alpha r$, is given by

$$
\lambda_{c}(k)=\lambda_{c} \frac{\left(9 \lambda_{c} \pi \alpha^{2} r^{2} / 4\right)^{k}}{k !} e^{-\alpha^{2} \lambda_{c} \pi r^{2}}
$$

where $\lambda_{c}$ and $\lambda_{c}(k)$ are the densities of sensing disks and covered k-components, respectively, and $\alpha r$ is the radius of theirs base circles at critical percolation.

Proof. Let $N$ be the total number of sensing sectors that are randomly deployed on a circular field of radius $\mathfrak{R}$ according to a Poisson point process with density equal to

$$
\lambda=\frac{N}{\pi \Re^{2}}
$$

Using $\omega_{k}=\lambda_{c}(k) \pi \Re^{2}$, which represents the mean number of covered k-components in the circular field, and equation (16) leads to

$$
\lambda_{c}(k)=\lambda \frac{\omega_{k}}{N}
$$

We can approximate $\frac{\omega_{k}}{N}$ by the probability $P\left[\operatorname{radius}\left(C_{k}^{c o v}\right)=\alpha r\right]$ of finding a covered k-component in a base circle whose radius is equal to $\alpha r$ and $\alpha$ will achieve from equation (14). Hence, we have

$$
P\left[\operatorname{radius}\left(C_{k}^{\text {cov }}\right)=\alpha r\right]=\frac{\omega_{k}}{N}
$$

Substituting (18) in (17) gives

$$
\lambda_{c}(k)=\lambda P\left[\operatorname{radius}\left(C_{k}^{c o v}\right)=\alpha r\right]
$$

By using definition $13, P\left[\operatorname{radius}\left(C_{k}^{\text {cov }}\right)=\alpha r\right]$ is the conditional probability of finding $\mathrm{k}$ sensing sectors enclosed in a circle with radius $\alpha r$ and centered at $\xi\left(C_{k}^{c o v}\right)$. Substituting $R_{k}=\alpha r$ into equation (4) gives

$$
P(k)=\frac{\left(9 \lambda_{c} \pi \alpha^{2} r^{2} / 4\right)^{k}}{k !} e^{-\alpha^{2} \lambda_{c} \pi r^{2}}
$$

and, hence, (19) becomes 


$$
\lambda_{c}(k)=\lambda_{c} \frac{\left(9 \lambda_{c} \pi \alpha^{2} r^{2} / 4\right)^{k}}{k !} e^{-\alpha^{2} \lambda_{c} \pi r^{2}}
$$

where $\lambda_{c}$ and $\lambda_{c}(k)$ are the critical densities of sensing sectors and covered k-components, respectively, $\alpha r$ is the radius of theirs base circles at critical percolation.

\subsubsection{Finding the Critical Percolation}

Now, we generate an equation that characterizes a set of covered k-components at critical percolation. By equating (11) and (15), we obtain a new equation $g\left(\lambda_{c}, r, \alpha, k\right)=0$, where

$$
g\left(\lambda_{c}, r, \alpha, k\right)=\left(\operatorname{erf}\left(\alpha \sqrt{\lambda_{c} \pi} r\right)-\alpha^{2} \sqrt{\lambda_{c}} r e^{-\alpha^{2} \lambda_{c} \pi r^{2}}\right)^{2} \times \frac{\left(9 \lambda_{c} \pi \alpha^{2} r^{2} / 4\right)^{k}}{k !} e^{-\alpha^{2} \lambda_{c} \pi r^{2}}-1
$$

By using definition 8 , we can rewrite the function $g$ as a function without direct dependence on $r$.

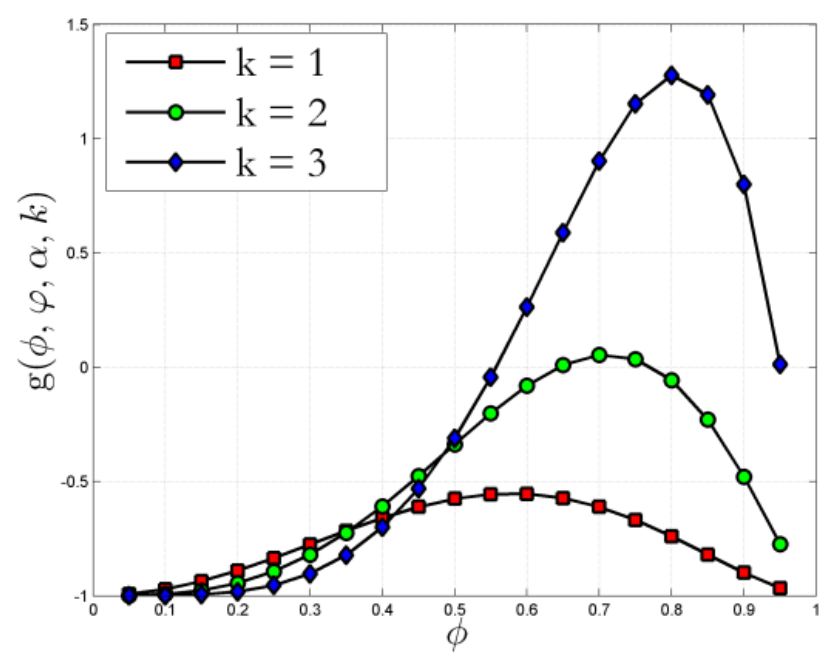

Fig. 12. Plot of function $g(\phi, \varphi, \alpha, k)$ for $\varphi=\pi, \alpha=1.0$ and $k=1,2$ and 3.

Percolation occurs in $\phi=0.644(\eta=1.032825)$ for $k=2$.

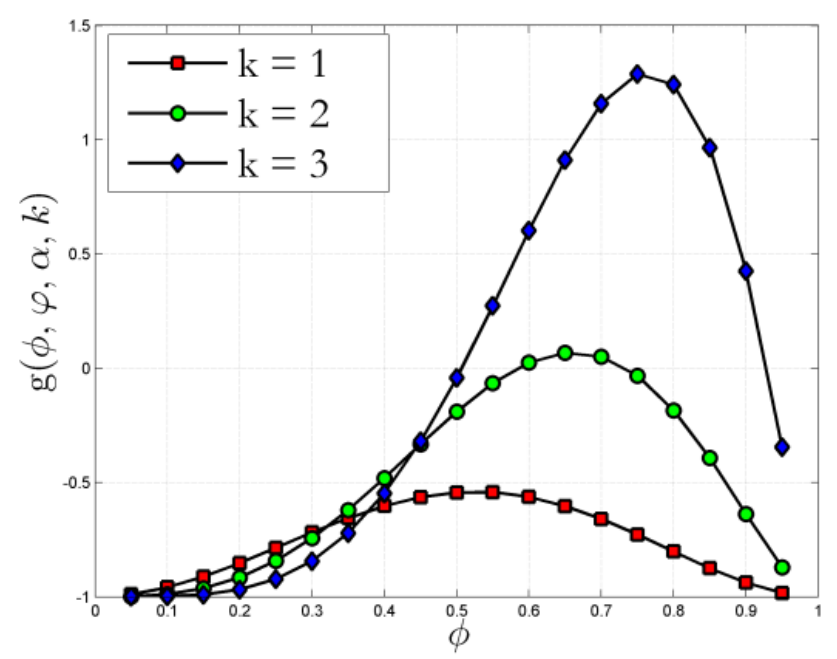

Fig. 13. Plot of function $g(\phi, \varphi, \alpha, k)$ for $\varphi=3 \pi / 4, \alpha=0.9239$ and $k=1,2$ and 3 .

Percolation occurs in $\phi=0.585(\eta=0.8794768)$ for $\mathrm{k}=2$. 


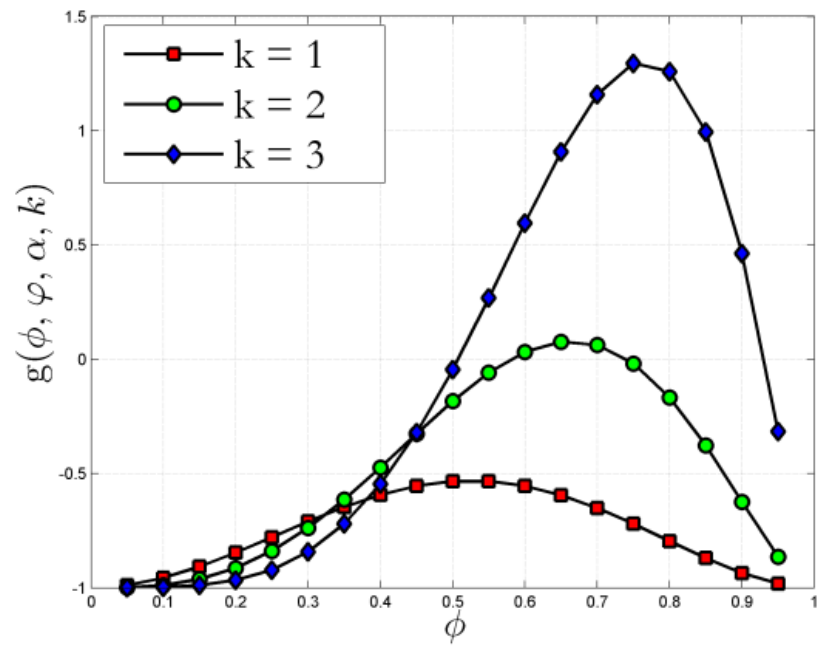

Fig. 14. Plot of function $g(\phi, \varphi, \alpha, k)$ for $\varphi=2 \pi / 3, \alpha=0.8660$ and $k=1,2$ and 3. Percolation occurs in $\phi=0.580(\eta=0.8675006$ for $k=2$.

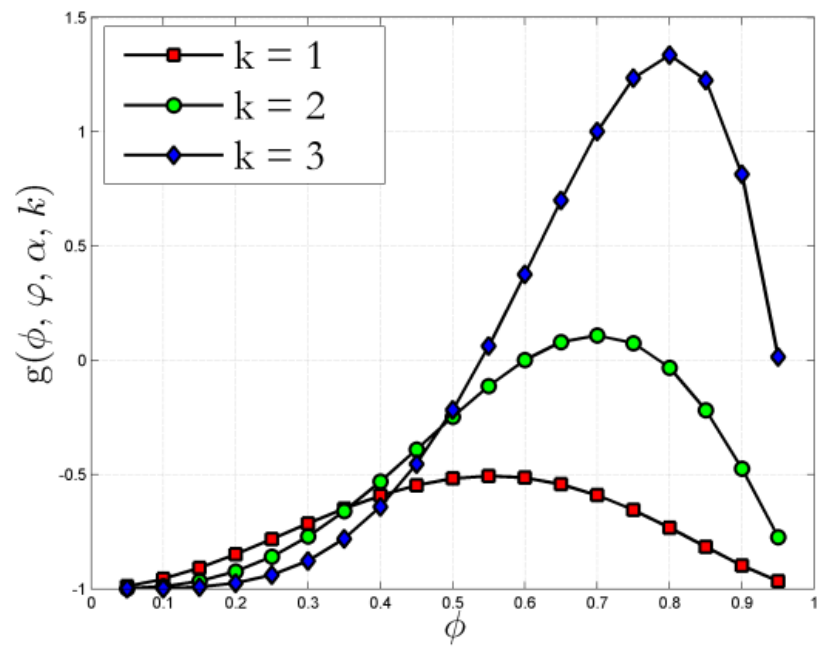

Fig. 15. Plot of function $g(\phi, \varphi, \alpha, k)$ for $\varphi=\pi / 2, \alpha=0.7071$ and $k=1,2$ and 3 . Percolation occurs in $\phi=0.600(\eta=0.9162907)$ for $k=2$.

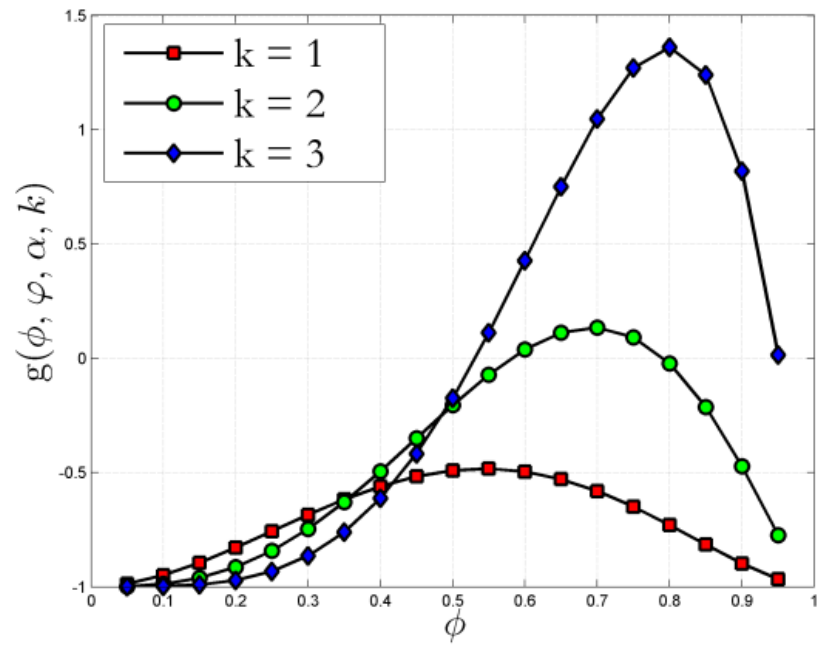

Fig. 16. Plot of function $g(\phi, \varphi, \alpha, k)$ for $\varphi=\pi / 3, \alpha=0.5$ and $k=1,2$ and 3 . Percolation occurs in $\phi=0.676(\eta=1.127012)$ for $k=2$. 


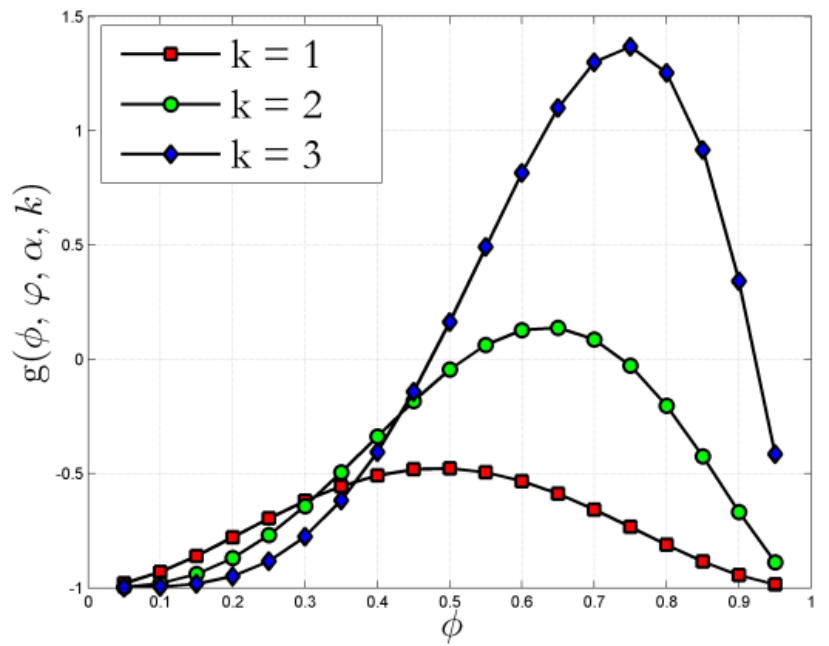

Fig. 17. Plot of function $g(\phi, \varphi, \alpha, k)$ for $\varphi=\pi / 4, \alpha=0.5000$ and $k=1,2$ and 3 . Percolation occurs in $\phi=0.570(\eta=0.8439701)$ for $k=2$.

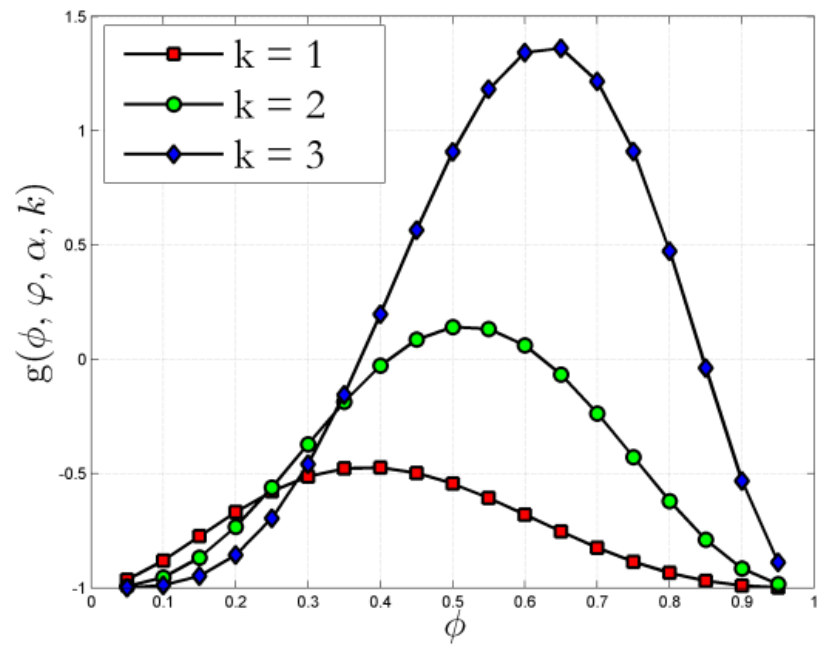

Fig. 18. Plot of function $g(\phi, \varphi, \alpha, k)$ for $\varphi=\pi / 6, \alpha=0.5000$ and $k=1,2$ and 3 . Percolation occurs in $\phi=0.431(\eta=0.5638748)$ for $k=2$.

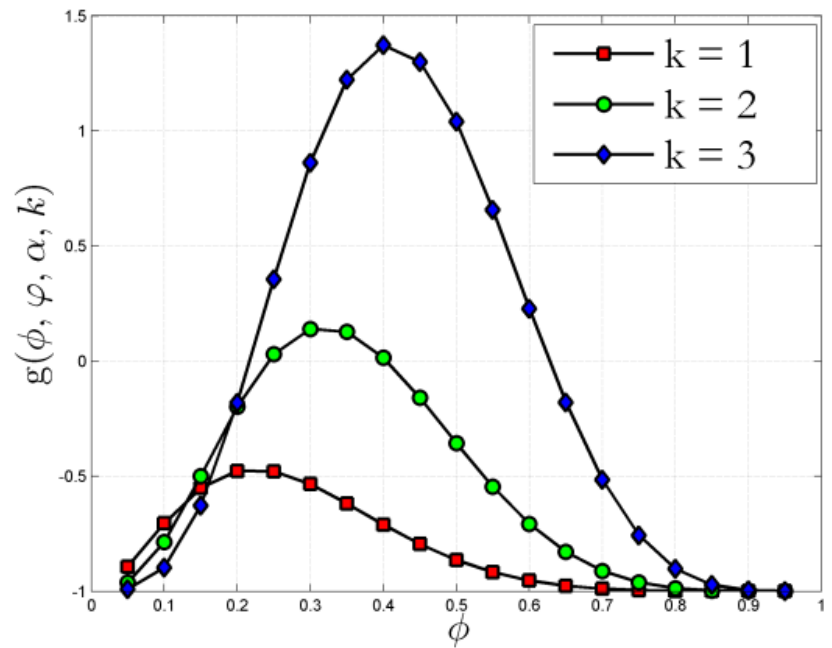

Fig. 19. Plot of function $g(\phi, \varphi, \alpha, k)$ for $\varphi=\pi / 12, \alpha=0.5000$ and $k=1,2$ and 3 . Percolation occurs in $\phi=0.246(\eta=0.2823629)$ for $k=2$. 


$$
g(\mu, \alpha, k)=\left(\operatorname{erf}(\alpha \sqrt{\mu})-\frac{\alpha^{2} \sqrt{\mu} e^{-\alpha^{2} \mu}}{\sqrt{\pi}}\right)^{2} \times \frac{\left(9 \alpha^{2} \mu / 4\right)^{k}}{k !} e^{-\alpha^{2} \mu}-1
$$

where $\mu$ is given by

$$
\mu=\lambda_{c} \pi r^{2}
$$

Because $a(\varphi)=\frac{1}{2} \varphi r^{2}$ is the area of sector based on its angle $(\varphi)$ [36], and also by using definition 8 we have

$$
\mu=\lambda_{c} \cdot\left(\frac{1}{2} \varphi r^{2}\right) \cdot\left(\frac{2 \pi}{\varphi}\right)=\eta_{c} \cdot\left(\frac{2 \pi}{\varphi}\right)
$$

where $\eta_{c}$ is the density of sectors at percolation. Because the filling factor has a definite range $(\phi \in[0,1])$, we use the definition 8 again to rewrite the equation (23) as

$$
\mu=-\log (1-\phi) \cdot\left(\frac{2 \pi}{\varphi}\right)
$$

Finally, the equation (21) could be rewritten as

$$
g(\phi, \varphi, \alpha, k)=\left(\operatorname{erf}(\alpha \sqrt{\mu})-\frac{\alpha^{2} \sqrt{\mu} e^{-\alpha^{2} \mu}}{\sqrt{\pi}}\right)^{2} \times \frac{\left(9 \alpha^{2} \mu / 4\right)^{k}}{k !} e^{-\alpha^{2} \mu}-1
$$

where $\mu$ is given by equation (24).

\subsection{Numerical Results}

Fig. 12 to Fig. 19 show the plots of equation (25) for $k=1,2$ and 3 . As it is seen, the function $g$ reaches to 0 from $k=2$ for all angles. It means that percolation occurs when $k \geq 2$ directional sensors exist in base circles. Table 3 presents $\eta$ and $\phi$ of field-of-view angles of table 1 and 2 that have been derived by using equation (25) and plots of Fig. 12 to Fig. 19.

Table 3

$\eta$ and $\phi$ for Eight Different Field-of-View Angles

\begin{tabular}{ccc}
\hline \hline$\varphi$ & $\eta_{c}$ & $\phi_{c}$ \\
\hline$\pi$ & 1.0328250 & 0.644 \\
$3 \pi / 4$ & 0.8794768 & 0.585 \\
$2 \pi / 3$ & 0.8675006 & 0.580 \\
$\pi / 2$ & 0.9162907 & 0.600 \\
$\pi / 3$ & 0.8698844 & 0.581 \\
$\pi / 4$ & 0.7318880 & 0.519 \\
$\pi / 6$ & 0.5293291 & 0.411 \\
$\pi / 12$ & 0.2770719 & 0.242 \\
\hline \hline
\end{tabular}




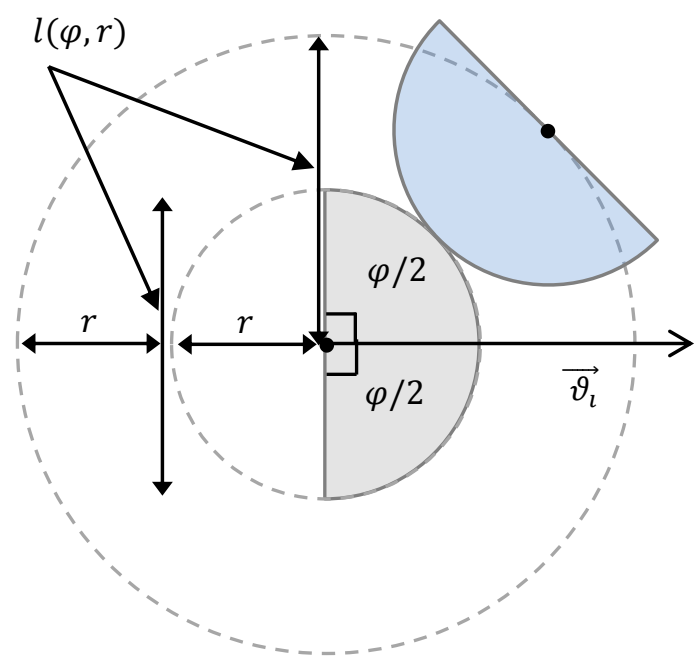

Fig. 20. Maximum distance of two collaborating sensors when $\varphi=\pi$.

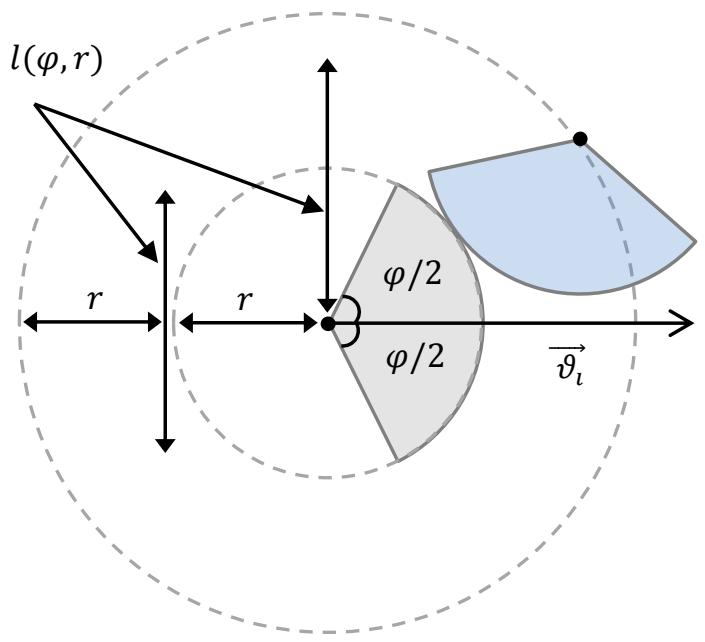

Fig. 21. Maximum distance of two collaborating sensors when $\varphi=3 \pi / 4$.

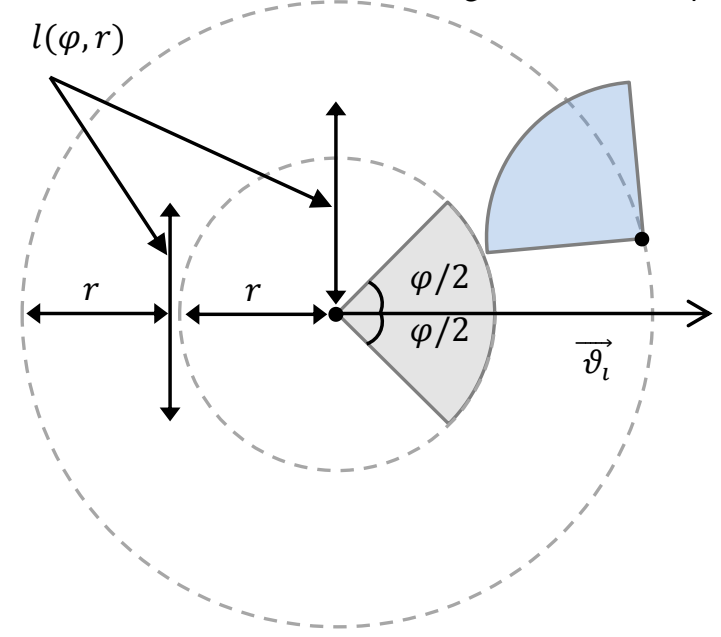

Fig. 22. Maximum distance of two collaborating sensors when $\varphi=\pi / 2$. 


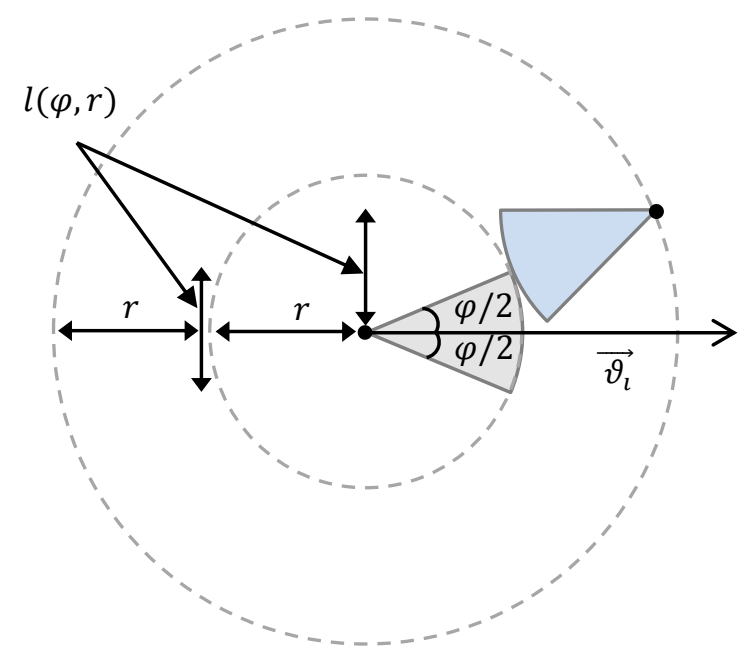

Fig. 23. Maximum distance of two collaborating sensors when $\varphi=\pi / 4$.

\section{Critical Density for Network Connectivity in FIODSNs}

Let $X_{\lambda}=\left\{\xi_{i}: i \geq 1\right\}$ be a two-dimensional homogeneous Poisson point process of density $\lambda$ (the number of sensors per unit area) and connection function $h_{C O N}$ (equation (6)), where $\xi_{i}$ represents the location of the sensor $S_{i}$. The NCPT problem can be expressed as "given a network that is initially disconnected, what is the probability of the first appearance of an infinite (or single large) connected component that spans the entire network?" As defined in definition 7, The NCPT problem requires that sensors be at a distance of at most $R$ from each other. Therefore, two sensors belong to the same communicated component, if $R$ (the radii of the transmission disks) of the sensors are at least equal to greatest distance between nodes. Next, we study the SCPT and NCPT problems together using percolation theory [8].

\section{Integrated Sensing Coverage and Network Connectivity in FIODSNs}

In this section, we consider a correlated model of sensor nodes to discuss on critical density. In this model, each sensor is associated with a disk with radius $R$ and a sector with radius $r$ and field-of-view angle $\varphi$ which are concentric. This kind of structure reveals a double behavior of the sensors that can be described by their collaboration and communication. The collaboration between sensors depends on the relationship between the radii of their sensing sectors and also the field-of-view angle, whereas communication is related to the relationship between the radii of their transmission disks [8].

As the most accurate findings, in [27], it has been shown that the percolation for disk shape objects (i.e. $R=2 r$ ) occurs in $\phi=0.67634831$ by using Monte Carlo method. Also, Ammari et al. [6] analytically has been shown that for $R=r$, percolation occurs in $\phi=0.925$ and for $R=1.25 r$, it occurs in $\phi=0.760$. Because filling factor for sensing coverage of directional sensor networks with field-of-view angle between 0 and $\pi$ (according to table 3 ) are less than disk shape objects, it is not reasonable to increase the density of sensors to achieve the network connectivity. Instead, we must consider the transmission range of sensors to be sure that network connectivity will be established too, in calculated densities by equation (25). Therefore, we focus on transmission radius when $r \leq R \leq 2 r$. In other words, we want to find the coefficient $\beta$ when $R=\beta r, 1 \leq \beta \leq 2$.

Connectivity between two sensors will be established when distance between them is less than or equal to their transmission range $(R)$. Due to randomness of sensors orientation in FIODSNs, we must consider the worst case of collaborating sensors (sensors which build the spanning cluster) for connectivity. As seen in Fig. 20 to Fig. 23, the maximum distance of two collaborating sensors in FIODSNs is equal to $2 r$ regardless of their field-of-view angle. In other words, to be sure that network connectivity between sensors 
of the infinite covered component is established too, the transmission range $(R)$ of sensors must be set to $2 r$. Therefore, $\beta$ must be equal to 2 .

Therefore, in a FIODSN with critical density of nodes for sensing coverage as calculated by equation (25), when $R \geq 2 r$ almost surely network connectivity percolates too.

\section{Simulations}

To examine the calculated critical densities, we simulated the percolation phase transition for all eight different field-of-view angles of table 3. As [8], we set the size of simulation environment to a square $50 \times$ 50 , sensing radius $(r)$ of sensors to 5 and transmission radius of sensors to $R=2 r$. The density interval for simulation has been set to 0.1 and the simulation repeated 500 times for each step. The percolation charts of SCPT and NCPT have been shown in Fig. 24. In all charts, the vertical line shows the calculated critical density for corresponding $\varphi$ by using equation (25).

As seen, in all charts, the percolation for sensing coverage almost surely occurs on or very close to calculated critical densities which are reasonable for an analytical and general equation. Also, as seen, network connectivity will be maintained almost surely for all different $\varphi$ on or after calculated critical densities.

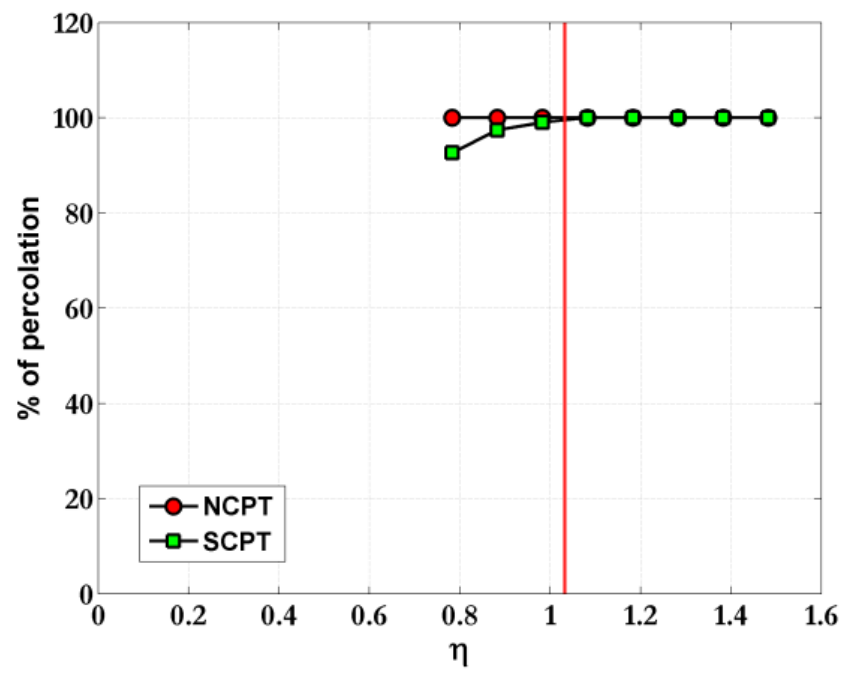

Fig. 24. Plot of simulation results for FIODSNs when $\varphi=\pi$.

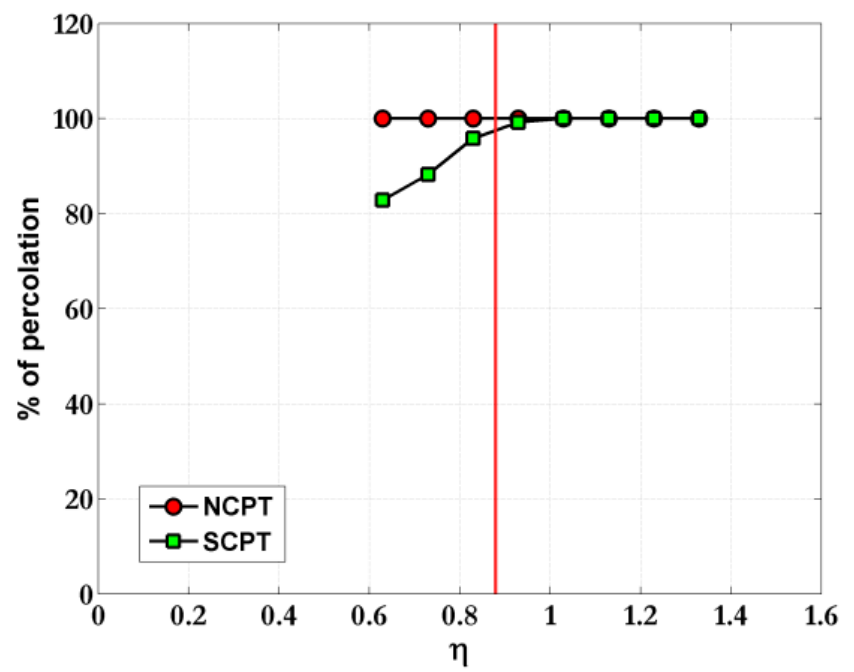

Fig. 25. Plot of simulation results for FIODSNs when $\varphi=3 \pi / 4$. 


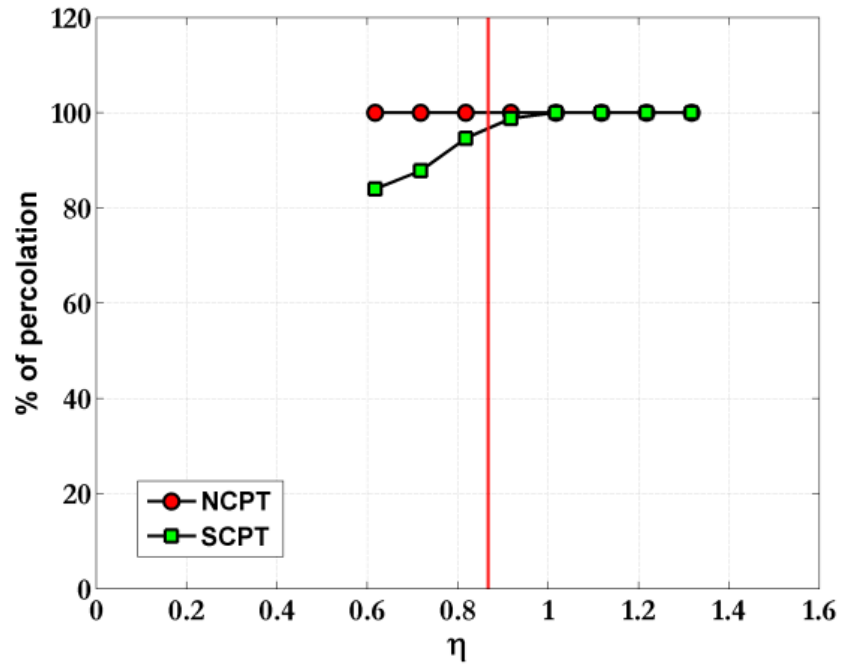

Fig. 26. Plot of simulation results for FIODSNs when $\varphi=2 \pi / 3$.

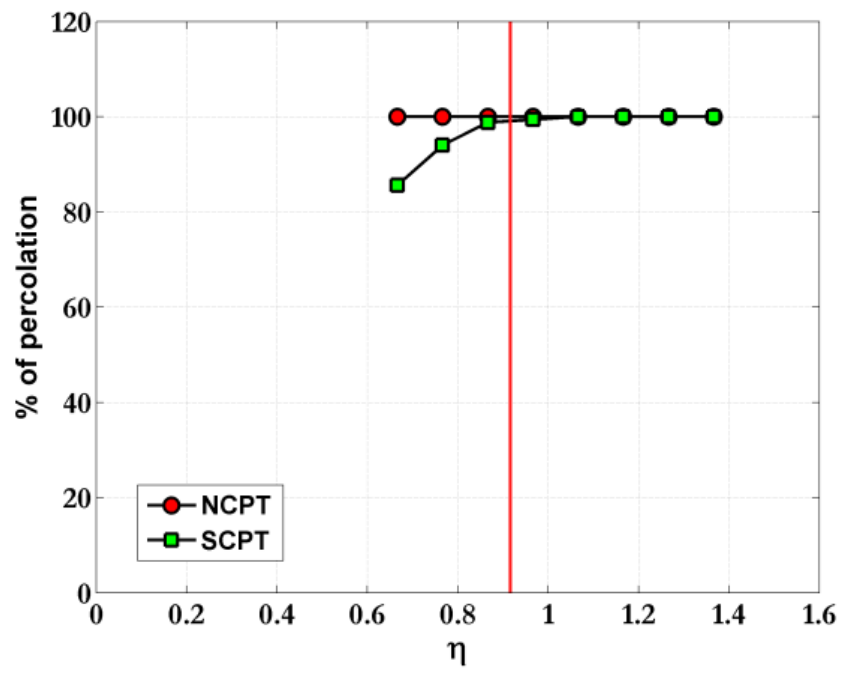

Fig. 27. Plot of simulation results for FIODSNs when $\varphi=\pi / 2$.

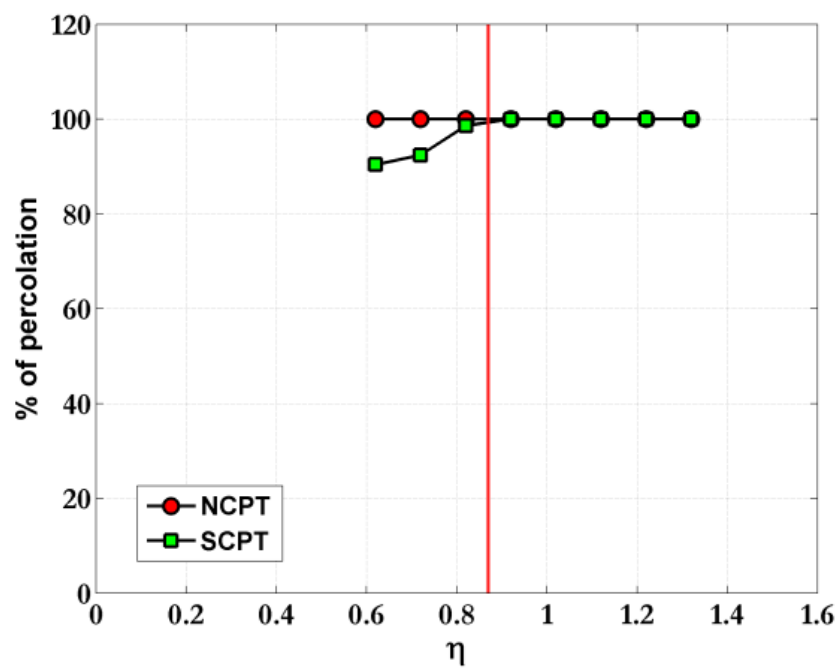

Fig. 28. Plot of simulation results for FIODSNs when $\varphi=\pi / 3$. 


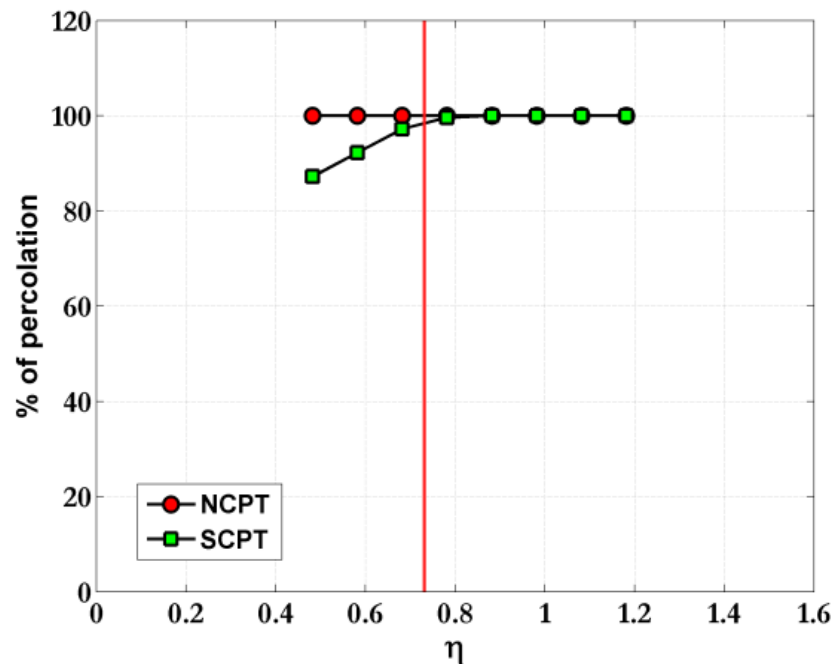

Fig. 29. Plot of simulation results for FIODSNs when $\varphi=\pi / 4$.

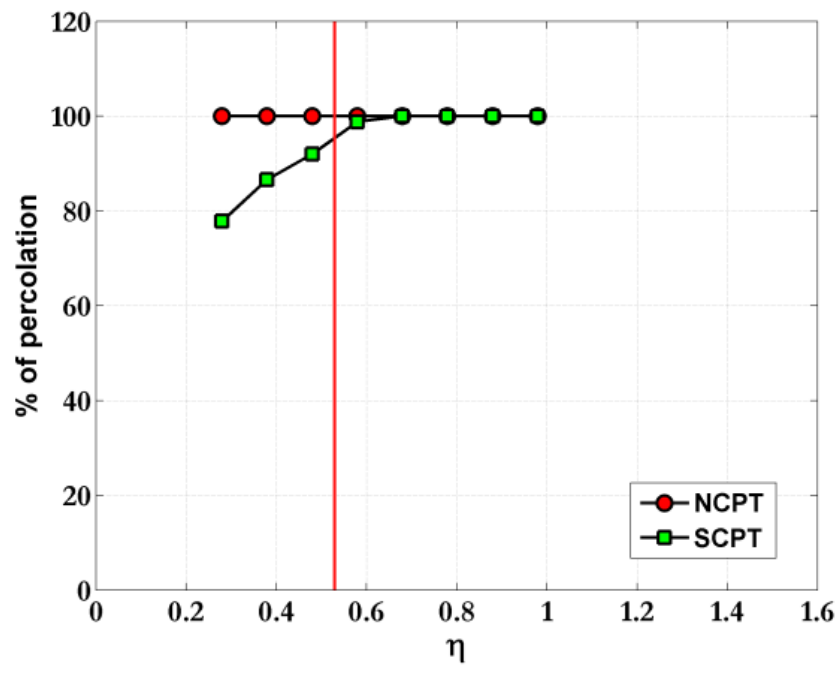

Fig. 30. Plot of simulation results for FIODSNs when $\varphi=\pi / 6$.

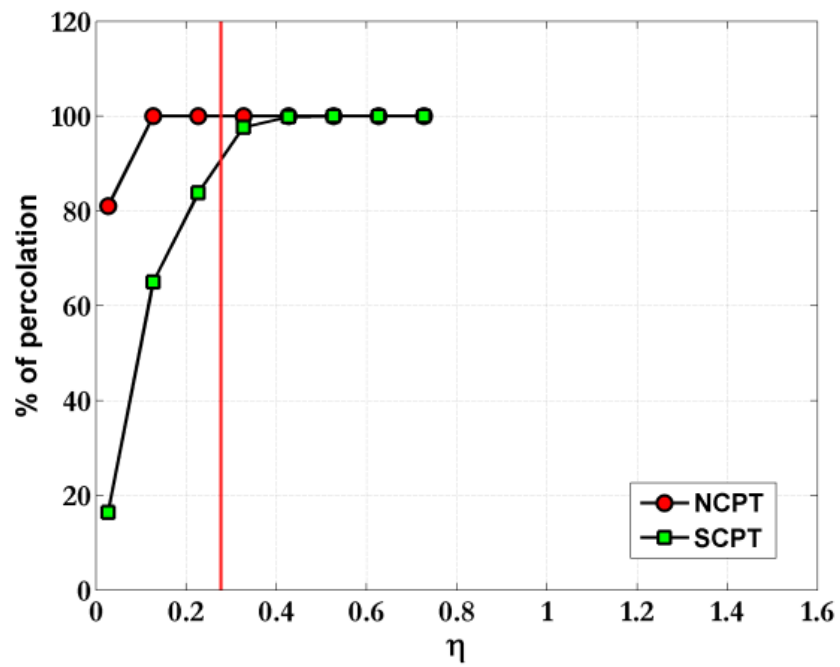

Fig. 31. Plot of simulation results for FIODSNs when $\varphi=\pi / 12$. 


\section{Conclusion}

In this paper, we considered fixed-orientation directional sensor networks (FIODSNs) in which nodes are deployed based on Poisson Point Process and the orientation of sensor nodes is distributed on $[0,2 \pi]$ independently and uniformly. Then, we proposed an approach to compute the critical density for sensing coverage and network connectivity in such networks, for all field-of-view angles between 0 and $\pi$ by using continuum percolation. The simulations confirmed that percolation occurs on or very close to the estimated critical densities which are reasonable for a general and analytical approach.

We also proposed a model for percolation in directional sensor network which provides a basis for solving the problem of finding critical density of nodes for SCPT and NCPT together. We proposed a correlated concentric disk and sector model in order to study SCPT and NCPT problems in directional sensor networks in an integrated way from a continuum percolation perspective. Precisely, we have considered the physical correlation between them, which is based on the ratio of the radius of the transmission disks of the sensors to the radius and field-of-view angle of their sensing sectors. Thus, when an infinite covered component arises for the first time, an infinite connected component will almost surely appear based on the ratio $2 \leq R / r$.

As future works, we are working on finding critical density of nodes in adjustable-orientation directional sensor networks (ADODSNs) in which the deployment is such as FIODSNs but the orientation of sensing sectors could be adjusted after deployment by using a distributed algorithm. Other possible future works include directional sensor networks with directional transmission antenna instead of common disk model, inhomogeneous directional sensor networks and three-dimensional directional sensor networks.

\section{REFERENCES}

[1] A. Ghosh, S. K. Das, Coverage and connectivity issues in wireless sensor networks: A survey, Pervasive Mob. Comput., vol. 4, no. 3, pp. 303-334, 2008.

[2] B. Wang, Coverage problems in sensor networks: A survey, ACM Comput. Surv., vol. 43, no. 4, article no. 32, 2011.

[3] C. Zhu, C. Zheng, L. Shu, G. Han, A survey on coverage and connectivity issues in wireless sensor networks, J. Netw. Comput. Appl., vol. 35, no. 2, pp. 619-632, 2012.

[4] M. A. Guvensan, A. G. Yavuz, on coverage issues in directional sensor networks: A survey, Ad Hoc. Netw., vol. 9, no. 7, pp. 1238-1255, 2011.

[5] Y. Charfi, N. Wakamiya, M. Murata, Challenging issues in visual sensor networks, IEEE Wirel. Commun., vol. 16, no. 2, pp. 44-49, 2009.

[6] H. M. Ammari, S. K. Das, Integrated coverage and connectivity in wireless sensor networks: A twodimensional percolation problem, IEEE T. Comput., vol. 57, no. 10, pp. 1423-1434, 2008.

[7] H. M. Ammari, S. K. Das, Critical density for coverage and connectivity in three-dimensional wireless sensor networks using continuum percolation, IEEE T. Parall. Distr., vol. 20, no. 6, pp. 872885, 2009.

[8] M. Khanjary, M. Sabaei, M.R. Meybodi, Critical density for coverage and connectivity in twodimensional aligned-orientation directional sensor networks using continuum percolation, IEEE Sens. J., vol. 14, no. 8, pp. 2856-2863, 2014.

[9] Y. Morsly, N. Aouf, M.S. Djouadi, M. Richardson, Particle swarm optimization inspired probability algorithm for optimal camera network placement, IEEE Sens. J., vol. 12, no. 5, pp. 1402-1412, 2012.

[10] J. Wang, C. Niu, R. Shen, Priority-based target coverage in directional sensor networks using a genetic algorithm, Comput. Math. Appl., vol. 57, no. 11-12, pp. 1915-1922, 2009.

[11] H. Mohamadi, S. Salleh, M. N. Razali, Heuristic methods to maximize network lifetime in directional sensor networks with adjustable sensing ranges, J. Netw. Comput. Appl., vol. 46, pp. 26$35,2014$. 
[12] M. Hooshmand, S. M. R. Soroushmehr, P. Khadivi, S. Samavi, S. Shirani, Visual sensor network lifetime maximization by prioritized scheduling of nodes, J. Netw. Comput. Appl., vol. 36, no. 1, pp. 409-419, 2013.

[13] Y. C. Tseng, P.Y. Chen, W. T. Chen,, K-angle object coverage problem in a wireless sensor network, IEEE Sens. J., vol. 12, no. 12, pp. 3408-3416, 2012.

[14] L. Liu, X. Zhang, H. Ma, Localization-oriented coverage in wireless camera sensor networks, IEEE T. Wirel. Commun., vol. 10, no. 2, pp. 484-494, 2011.

[15] X. Han, X. Cao, E. L. Lloyd, C. C. Shen, Deploying directional sensor networks with guaranteed connectivity and coverage, in: Proceedings of Fifth Annual IEEE Communications Society Conference on Sensor, Mesh and Ad Hoc Communications and Networks (SECON), 2008, pp. 153160.

[16] U. R. Chen, B. S. Chiou, J. M. Chen, W. Lin, An adjustable target coverage method in directional sensor networks, in: Proceedings of 3rd IEEE Asia-Pacific Services Computing Conference (APSCC), 2008, pp. 174-180.

[17] Y. Wang, G. Cao, on full-view coverage in camera sensor networks, in: Proceedings of 30th IEEE International Conference on Computer Communications (INFOCOM), 2011, pp. 1781-1789.

[18] E. N. Gilbert, Random Plane Networks, J. SIAM, vol. 9, no. 4, pp. 533-543, 1961.

[19] I. Glauche, W. Krause, R. Sollacher, M. Greiner, Continuum percolation of wireless ad hoc communication networks, Physica A, vol. 325, pp. 577-600, 2003.

[20] A. Jiang, J. Bruck, Monotone percolation and the topology control of wireless networks, in: Proceedings of 24th IEEE International Conference on Computer Communications (INFOCOM), 2005, pp. 327-338.

[21] F. Xing, W. Wang, on the critical phase transition time of wireless multi-hop networks with random failures, in: Proceedings of 14th Annual International Conference on Mobile Computing and Networking (MOBICOM), 2008, pp. 175-186.

[22] L. Liu, X. Zhang, H. M. Ma, Optimal density estimation for exposure-path prevention in wireless sensor networks using percolation theory, in: Proceedings of 31st IEEE International Conference on Computer Communications (INFOCOM), 2012, pp. 2601-2605.

[23] L. Liu, X. Zhang, H. Ma, Percolation theory-based exposure-path prevention for wireless sensor networks coverage in internet of things, IEEE Sens. J., vol. 13, no. 10, pp. 3625-3636, 2013.

[24] L. Liang, Z. Xi, M. Huadong, Exposure-path prevention in directional sensor networks using sector model based percolation, in: Proceedings of 44th IEEE International Conference on Communications, (ICC), 2009, pp. 1-5.

[25] G. Yang, D. Qiao, Critical conditions for connected-k-coverage in sensor networks, IEEE Commun. Lett., vol. 12, no. 9, pp. 651-653, 2008.

[26] P. Balister, Z. Zheng, S. Kumar, P. Sinha, Trap coverage: allowing coverage holes of bounded diameter in wireless sensor networks, in: Proceedings of 28th IEEE International Conference on Computer Communications (INFOCOM), 2009, pp. 136-144.

[27] S. Mertens, C. Moore, Continuum percolation thresholds in two dimensions, Phys. Rev. E, vol. 86, $061109,2012$.

[28] R. Meester, R. Roy, Continuum percolation, Cambridge University Press, 1996.

[29] P. Hall, Introduction to the theory of coverage processes, John Wiley \& Sons, 1988.

[30] S. R. Broadbent, J. M. Hammersley, Percolation Processes: I. Crystals and Mazes, Proc. Cambridge Philosophical Soc., vol. 53, pp. 629-641, 1957.

[31] G. Grimmett, Percolation, Springer Verlag, 1989.

[32] J. W. Essam, Percolation theory, Reports on Progress in Physics, vol. 43, pp. 833-912, 1980.

[33] http://mathworld.wolfram.com/LawofCosines.html, 2014.

[34] N. A. C. Cressie, Statistics for spatial data, John Wiley \& Sons, 1991.

[35] http://mathworld.wolfram.com/Erf.html, 2014.

[36] http://mathworld.wolfram.com/CircularSector.html, 2014. 


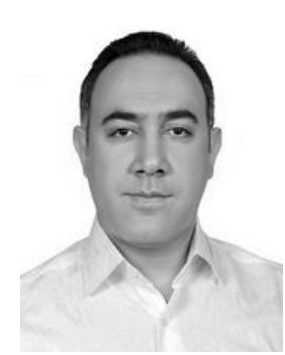

Mohammad Khanjary received his B.Sc. degree in computer engineering from Amirkabir University of Technology, Tehran, Iran and his M.Sc. degree from Qazvin Branch, Islamic Azad University, Qazvin, Iran in 2004 and 2009, respectively. Currently, $\mathrm{He}$ is Ph.D. student of computer engineering at Science and Research Branch, Islamic Azad University, Tehran, Iran. His current research interest includes computer networks and mathematical modelling.

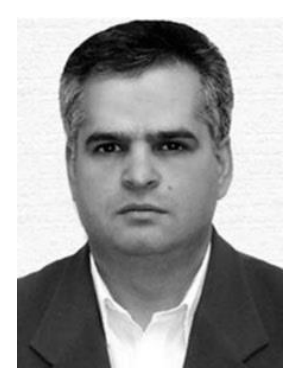

Masoud Sabaei received his B.Sc. degree from Esfahan University of Technology, Esfahan, Iran, and his M.Sc. and Ph.D. form Amirkabir University of Technology (Tehran Polytechnic), Tehran, Iran, all in the field of Computer Engineering in 1992, 1995 and 2000, respectively. Dr. Sabaei has been professor of Computer Engineering Department, Amirkabir University of Technology (Tehran Polytechnic), Tehran, Iran since 2002. His research interests are wireless networks, software defined networks, and telecommunication network management.

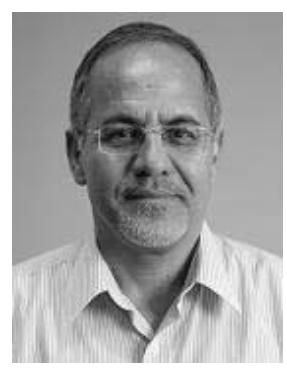

Mohammad Reza Meybodi received the BS and MS degrees in Economics from Shahid Beheshti University in Iran, in 1973 and 1977, respectively. He also received the MS and PhD degree from Oklahoma University, USA, in 1980 and 1983, respectively in Computer Science. Currently, he is a full professor in Computer Engineering Department, Amirkabir University of Technology, Tehran, Iran. Prior to his current position, he worked from 1983 to 1985 as an assistant professor at Western Michigan University, and from 1985 to 1991 as an associate professor at Ohio University, USA. His research interests include channel management in cellular networks, learning systems, parallel algorithms, soft computing and software development. 